\title{
Protein Kinase CK2 Controls the Stability of Prostate Derived ETS Factor
}

\author{
Cristine Chisholm, Achuth Padmanabhan, Bin Guan, Xiang Li, Simran Kalra and \\ Charles J. Bieberich*
}

Department of Biological Sciences, University of Maryland Baltimore County, Baltimore, MD, 21250, USA

\begin{abstract}
Prostate Derived ETS Factor $(P D E F)$ is an ETS transcription factor expressed in prostate epithelial cells, and diminished PDEF protein accumulation is correlated with prostate cancer progression. PDEF interacts with the tumor suppressor NKX3.1, and this interaction abolishes the ability of PDEF to activate the Prostate-Specific Antigen promoter. NKX3.1 stability is known to be regulated by Protein Kinase CK2 and the E3 ubiquitin ligase TOPORS. To determine if PDEF and NKX3.1 are coordinately regulated in prostate cancer cells, the effect of CK2 inhibition on steady-state PDEF levels was explored. Inhibition of CK2 activity with apigenin or 4,5,6,7-tetrabromo-benzimidazole (TBB) reduced steadystate levels of PDEF in LNCaP cells, and this effect was reversed by inhibiting the 26S proteasome. siRNA-mediated knockdown of $\mathrm{CK} 2 \alpha^{\prime}$ in LNCaP cells also reduced PDEF accumulation. Mass spectrometric analysis of phosphorylated recombinant PDEF revealed that Thr144, Ser151 and Ser187 are CK2 phosphoacceptor sites in vitro. PDEF was also robustly polyubiquitinated by TOPORS in vitro. These results suggest that PDEF and NKX3.1 are coordinately regulated by CK2 phosphorylation that inhibits their proteasomal degradation in prostate cancer cells.
\end{abstract}

Keywords: PDEF, CK2, TOPORS, TBB, NKX3.1, ETS, Ubiquitination.

\section{INTRODUCTION}

The ETS transcription factors influence a diverse array of cellular processes, including organogenesis, angiogenesis, cell cycle control, cell proliferation and motility, extracellular matrix remodeling, and hematopoietic differentiation. ETS factors have characteristics of both oncogenes and tumor suppressors [1]. The altered or aberrant expression of ETS factors in prostate epithelium can directly contribute to the development and progression of carcinoma [2]. ETS factors are controlled by posttranslational modification(s), particularly phosphorylation [3, 4]. A well-characterized example is the regulation of several subfamilies of ETS proteins by the Ras Mitogen-Activated Protein Kinase (MAPK) pathway where selective phosphorylation by MAPKs controls the ability to activate transcription of target genes, which in turn may contribute to the initiation, maintenance, or progression of neoplasia [3].

Prostate Derived ETS Factor (PDEF) is a member of the epithelial-specific ETS factor subfamily and is expressed in epithelial cells of the prostate, mammary, and salivary glands, colon, and ovary. Modulating PDEF expression in prostate, breast and colon cancer cells has revealed roles for this protein in controlling cell migration and invasion [5-10]. Constitutive or inducible PDEF expression inhibits migration and invasion in multiple breast cancer lines, and concomitant changes in focal adhesion complexes [8]. Consistent with these observations, knockdown of PDEF in prostate cancer cells results in increased migration and invasiveness and

*Address correspondence to this author at the Department of Biological Sciences, University of Maryland, Baltimore County, Baltimore, MD 21250, USA; Tel: (410) 455-3125; Fax: (410) 455-3875. E-mail: bieberic@umbc.edu changes in expression of genes involved in these processes [5]. In contrast, however, PDEF overexpression in breast cancer cells results in an invasive and promigratory phenotype in the context of deregulated ERK/MAPK and constitutively active ErbB2/CSF-1R [6]. This apparent paradox may point to a context-dependent role of PDEF, and highlights the potential impact of altered signaling pathways.

PDEF protein expression in human breast and prostate cancers has been explored using a series of independently derived polyclonal antisera. Consistent with an anti-migration/invasion function, Feldman et al. demonstrated diminished PDEF accumulation in six of seven invasive ductal breast carcinoma cases [9]. In contrast, increased PDEF expression was observed in $90 \%$ of breast tumors in a tissue microarray [11]. Similarly, both diminished [12] and increased [11] PDEF accumulation has been reported in prostate cancer cases. Given the extent of morbidity and mortality associated with breast and prostate cancer, and the clear evidence of a role for this protein in controlling epithelial cells migration and invasion, it will be imperative to resolve these paradoxical observations.

A recent proteomic analysis identified 121 potential protein partners of PDEF in breast cancer cells [13]. Proteins involved in a variety of cellular processes and in multiple subcelluar compartments were revealed, and interaction with Catenin gamma-1 and the serine/threonine kinase p62 were confirmed by immunoprecipitation/Western blot analyses [13]. PDEF has been demonstrated to interact with key regulators of prostate epithelial differentiation, including Androgen Receptor (AR) and the prostate tumor suppressor NKX3.1 [14, 15]. PDEF interaction with AR potentiates androgen-mediated activation of the Prostate Specific Antigen promoter, and NKX3.1 abolishes this activity [15]. NKX3.1 is known to be regulated by Protein Kinase CK2, 
which protects NKX3.1 from proteasome-dependent degradation [16].

CK2 is a ubiquitous, constitutively active serine/threonine kinase with over 300 known substrates. The CK2 consensus phosphorylation site is $\mathrm{S} / \mathrm{TXXD} / \mathrm{E}$, where $\mathrm{X}$ is a non-basic residue, and the $n+3$ position relative to the target serine or threonine is most important [17]. CK2 is a member of the CMGC kinase subfamily whose members include Glycogen Synthase Kinase 3, cyclin-dependent kinases, and MAPKs [18]. Many transcription factors, for example tumor suppressor p53 and $\beta$-catenin, are targets of CK2 phosphorylation [19]. The CK2 holoenzyme exists as a heterotetramer, comprised of two $\beta$ subunits complexed with catalytic $\alpha$ and/or $\alpha^{\prime}$ subunits. The free catalytic $\alpha$ and $\alpha^{\prime}$ subunits are also active independent of the holoenzyme and are believed to have distinct and separate functions [20]. Although the overall activity of $\mathrm{CK} 2$ is elevated in prostate tumor cells compared with normal prostate epithelial cells, the subcellular location and amount of free and complexed catalytic subunits of the kinase are relevant to the resulting biology [21]. The loss of the free $\alpha^{\prime}$ catalytic subunit from the nucleus could contribute to reduced or lost expression of NKX3.1 in prostate cancer [16].

In light of the fact that CK2 phosphorylation prevents ubiquitin-mediated degradation of NKX3.1 by the $26 \mathrm{~S}$ proteasome, we postulated that $\mathrm{CK} 2$ could also potentially control PDEF stability $[14,16]$. Here, we demonstrate that CK2 phosphorylates PDEF in vitro and identify three CK2 phosphoacceptor sites. We further demonstrate that pharmacologic inhibition of CK2 significantly reduces the steadystate levels of PDEF in prostate cancer cells, and show that PDEF can be polyubiquitinated by an E3 ubiquitin ligase that regulates NKX3.1 turnover. Together, these data indicate that PDEF and NKX3.1 are coordinately regulated at the post-translational level in prostate cancer cells.

\section{MATERIALS AND METHODS}

\section{Development and Validation of a Rabbit Polyclonal Anti- PDEF Antibody}

N-terminal amino acids 1-141 of human PDEF $\left(\mathrm{PDEF}^{1-141}\right)$ were cloned into pQE80L (Promega). Recombinant $\mathrm{N}$-terminal $6 \mathrm{xHis}-\mathrm{PDEF}$ protein was induced in $E$. coli strain BL21 at $37{ }^{\circ} \mathrm{C}$ with $0.6 \mathrm{mM}$ isopropylthiogalactopyranoside. Cells were pelleted, resuspended in $300 \mathrm{mM}$ $\mathrm{NaCl}, 50 \mathrm{mM} \mathrm{NaH} 2 \mathrm{PO}_{4}, 20 \mathrm{mM}$ imidazole, $\mathrm{pH} 7.5$, broken at $18,000 \mathrm{lb} / \mathrm{in}^{2}$ in a French press and centrifuged at 20,000 x g for 30 minutes at $4{ }^{\circ} \mathrm{C}$. The supernatant was collected and loaded to a nickel column (Ni-NTA (Qiagen)). PDEF was eluted with $300 \mathrm{mM} \mathrm{NaCl}, 50 \mathrm{mM} \mathrm{NaH} \mathrm{PO}_{4}, 250 \mathrm{mM}$ imidazole, $\mathrm{pH}$ 7.5. Fractions containing purified PDEF were used to gene-rate polyclonal antibodies in a New Zealand White rabbit. Serum and terminal bleeds were affinity purified against $\mathrm{PDEF}^{1-141}$ and tested for reactivity by Western blot using recombinant PDEF protein and $\mathrm{LNCaP}$ cells transfected with full length human PDEF. Further determination of specificity and sensitivity was confirmed by Western blot comparisons with two commercially available anti-PDEF antibodies (Zymed and Santa Cruz), and Western blot analysis of protein signal in $\mathrm{LNCaP}$ cells treated with PDEF siRNA was compared with untreated LNCaP cells.

\section{In Vitro Kinase Assay}

In vitro kinase reactions were carried out in a buffer containing full-length human recombinant PDEF, CK2 holoenzyme (New England Biolabs) or recombinant CK2 $\alpha$, $200 \mu \mathrm{M}$ ATP, and 1X CK2 reaction buffer (New England Biolabs) at room temperature for 60 minutes in a $100 \mu 1$ volume.

\section{Cell Culture, Pharmacologic Inhibition of CK2, Western and Northern Blot Analysis}

LNCaP cells were maintained in RPMI-1640 plus $10 \%$ fetal bovine serum in a humidified incubator with $5 \% \mathrm{CO}_{2}$. Culture media was supplemented with apigenin $(75 \mu \mathrm{M})$ (Sigma), 4,5,6,7-tetrabromobenzotriazole (TBB) $(50 \mu \mathrm{M})$ (Sigma and Calbiochem), or MG132 (10.5 $\mu \mathrm{M})$ (Sigma) by dilution of 10 or $20 \mathrm{mM}$ stock solutions in dimethylsulfoxide (DMSO). At specific time points, cells were harvested by trypsinization and lysed in $150 \mathrm{mM} \mathrm{NaCl}, 50 \mathrm{mM}$ Tris, $0.25 \% \mathrm{NP}-40, \mathrm{pH} 7.5$, and protein concentration was determined using Bio-Rad protein assay reagent (Bio-Rad). Identical amounts of total protein were loaded on $12 \%$ SDSPAGE gels and after electrophoresis, were transferred to polyvinylidene difluoride membranes. Rabbit anti-PDEF was added at 1:1,000 dilution at $4{ }^{\circ} \mathrm{C}$ overnight. In parallel, whole RNA was extracted from apigenin-treated cells and DMSO vehicle controls using an RNeasy Kit according to the manufacturer's instructions (Qiagen), and separated on a $1 \%$ glyoxal/DMSO/agarose gel (Ambion) and hybridized to a PDEF cDNA probe.

\section{Mass Spectrometry}

Liquid chromatography-tandem mass spectrometry (LCMS/MS) was performed on CK2-phosphorylated recombinant PDEF that was resolved by 2D SDS-PAGE. Spots corresponding to phosphorylated PDEF were excised from a silver-stained gel. After in-gel trypsin digestion, eluted peptides were resolved on a $\mathrm{C} 18$ reverse phase column coupled on-line to an LTQ linear ion trap mass spectrometer (Thermo Fisher Scientific). $\mathrm{MS}^{3}$ was performed on precursor ions demonstrating neutral loss of a phosphate group. Data analysis was preformed using the Bioworks 3.3.1 software (Thermo Fisher Scientific).

\section{Expression Constructs and GST Pull-Downs}

Full-length recombinant PDEF was expressed and purified as described [22]. Recombinant GST-TOPORS was expressed and purified as described [23].

\section{In Vitro Ubiquitination Assay}

In vitro ubiquitination assays were carried out in $50 \mathrm{mM}$ HEPES, pH 7.9, $5 \mathrm{mM} \mathrm{MgCl} 2,15 \mu \mathrm{M} \mathrm{ZnCl}, 4 \mathrm{mM}$ ATP with the addition of $100 \mathrm{nM}$ rabbit E1 (Boston Biochem), $200 \mathrm{nM}$ human UbcH5a, and $250 \mu \mathrm{M}$ human wild type recombinant ubiquitin (Boston Biochem) or no-lysine ubiquitin (Boston Biochem). Reactions were carried out at 
$37{ }^{\circ} \mathrm{C}$ for 60 minutes in a $10 \mu 1$ volume. In vivo ubiquitination assays were performed as described [23].

\section{RESULTS}

\section{Production and Characterization of an Anti-PDEF Polyclonal Antiserum}

To reduce the likelihood of cross-reactivity with other ETS factors, the N-terminal 141 amino acids of human PDEF were chosen as the antigen (Fig. 1A). PDEF $^{1-141}$ does not bear significant homology to other ETS factors or other human proteins, however, there is $89 \%$ homology in this region between mouse and human PDEF. This region was cloned into the bacterial expression vector pEQ80L, and expressed in BL21 cells. The protein was enriched from bacterial lysates by nickel affinity and anion exchange chromatography to $\sim 90 \%$ purity. New Zealand White rabbits were immunized following a standard boost/bleed regimen, and post-immunization sera were screened by Western blot analysis of LNCaP cells transfected with exogenous PDEF (data not shown) and LNCaP cells treated with siRNA specific to PDEF (Fig. 1B). A prominent $46 \mathrm{kDa}$ species was observed, and was specifically diminished in the PDEF siRNA-treated cells. This apparent molecular weight is consistent with previous analyses of PDEF by SDS-PAGE $[9,10,24]$. Other molecular weight species were variably observed in Western blot analyses (Fig. 1B) however these were not diminished by treatment with PDEF siRNA and represent cross-reactivity of the polyclonal sera. To further verify the specificity of the anti-PDEF ${ }^{1-141}$, parallel Western blots with commercially available (Zymed, Santa Cruz Biotechnology), and existing anti-PDEF antibodies [8, 24] were performed (data not shown). This comparison indicated that our anti-PDEF antibody recognized a $46 \mathrm{kDa}$ isoform PDEF indistinguishable from that recognized by extant antibodies.

\section{Protein Kinase CK2 Phosphorylates PDEF}

To determine if PDEF can be phosphorylated by CK2, in vitro kinase reactions were carried out with full-length recombinant PDEF or the N-terminal domain (amino acids 1-141) with CK2 holoenzyme or CK2 $\alpha$ alone in the presence of $\gamma-{ }^{32} \mathrm{P}$-ATP. Robust phosphorylation was observed after gel electrophoresis followed by autoradiography (data not shown). To determine specific sites of CK2 phosphorylation in PDEF, in vitro kinase reactions were analyzed by LCMS/MS. Products of kinase reactions were resolved by twodimensional SDS-PAGE, silver stained, excised, and digested with trypsin. Mass spectra obtained from non-phosphorylated PDEF were compared with spectra generated from PDEF phosphorylated by CK2. These data revealed evidence for three CK2 phosphoacceptor sites in PDEF: Serine 187 , in a fragment spanning amino acids 182-192, ELCAMS*EEQFR, with a mass of 1342.5766, and Threonine 144 and Serine 151, in a fragment spanning amino

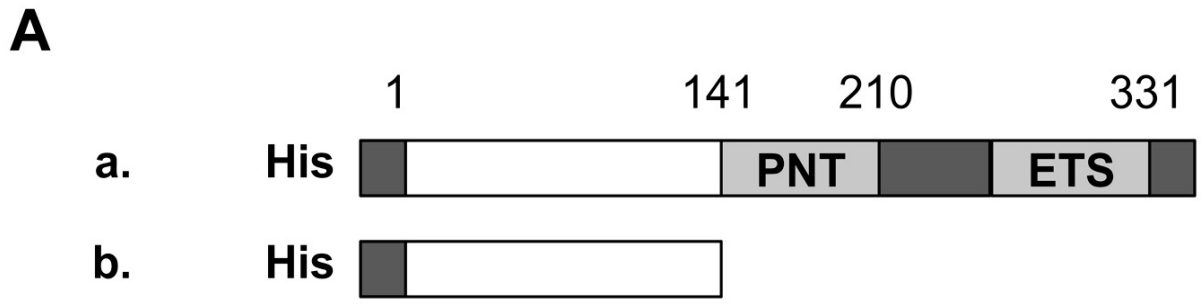

B

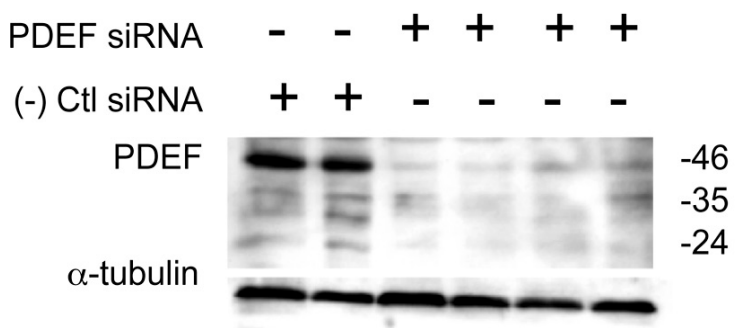

Fig. (1). Production of an anti-PDEF polyclonal antibody. A. The N-terminal region of PDEF (amino acids 1-141) was used to immunize rabbits to generate polyclonal anti-PDEF antibodies. B. Western blot detection of endogenous PDEF protein in LNCaP cells 24 hours after transfection with a PDEF siRNA or a negative control siRNA. The $46 \mathrm{kDa}$ species is significantly diminished specifically in the PDEF siRNA-treated lanes. The blots were stripped and probed with an antibody against $\alpha$-tubulin as a loading control. 
Table 1. Peptides Identified by Mass Spectrometric Analysis of Recombinant PDEF Phosphorylated in vitro by CK2

\begin{tabular}{|c|c|c|c|c|c|c|c|c|c|c|}
\hline Scans $^{1}$ & Peptide & MH+ & DeltaM & $\mathbf{z}$ & Type & $\begin{array}{l}\text { P(pro)/ } \\
\text { P(pep) }\end{array}$ & Sf & $\begin{array}{l}\text { Score } \\
\text { Xc }\end{array}$ & $\begin{array}{l}\text { Coverage } \\
\text { Delta Cn }\end{array}$ & $\begin{array}{c}\text { MW } \\
\text { Sp }\end{array}$ \\
\hline \multicolumn{6}{|c|}{ 1 SAM pointed domain containing ets transcription factor [Homo sapiens] } & $2.30 \mathrm{E}-13$ & 8.62 & 142.28 & & 37493.9 \\
\hline 4223 & K.AAAGAVGLER.R & 914.50543 & 0.46434 & 2 & CID & $3.84 \mathrm{E}-05$ & 0.94 & 3.54 & 0.41 & 947.6 \\
\hline 4305 & -AFQELAGK- & 863.46216 & 0.32945 & 2 & CID & $1.26 \mathrm{E}-03$ & 0.80 & 2.44 & 0.15 & 638.1 \\
\hline 4400 & K.AFQELAGK.E & 863.46216 & 0.47576 & 2 & CID & $1.20 \mathrm{E}-02$ & 0.90 & 2.70 & 0.31 & 710.9 \\
\hline 4929 & K.ELCAMSEEQFR.Q & 1342.57654 & 1.75798 & 2 & CID & $7.76 \mathrm{E}-07$ & 0.88 & 3.05 & 0.36 & 434.2 \\
\hline 5053 & -ELCAMS\#EEQFR- & 1324.57660 & 0.40455 & 2 & CID & $1.96 \mathrm{E}-02$ & 0.33 & 2.03 & 0.20 & 198.3 \\
\hline 5304 & -ELCAMSEEQFR- & 1342.57654 & -0.44455 & 2 & CID & $1.10 \mathrm{E}-06$ & 0.18 & 1.97 & 0.05 & 179.5 \\
\hline 5407 & -MGSASPGLSSVSPSHLLLPPDTVSR- & 2492.28125 & 1.69458 & 3 & CID & $1.87 \mathrm{E}-04$ & 0.30 & 2.78 & 0.15 & 291.0 \\
\hline $\begin{array}{l}5416- \\
5417\end{array}$ & R.SPLGGDVLHAHLDIWK.S & 1757.93335 & 1.19345 & 3 & CID & $2.90 \mathrm{E}-12$ & 0.93 & 3.76 & 0.42 & 1257.1 \\
\hline 5420 & -WLLWTEHQYR- & 1431.71680 & -0.95431 & 3 & CID & $2.36 \mathrm{E}-05$ & 0.32 & 1.69 & 0.13 & 395.9 \\
\hline 5482 & K.WLLWTEHQYR.L & 1431.71680 & 0.57964 & 2 & CID & $6.20 \mathrm{E}-07$ & 0.94 & 3.38 & 0.43 & 892.8 \\
\hline 5588 & -LLNITADPMDWSPSNVQK- & 2029.00586 & -0.40571 & 2 & CID & $8.92 \mathrm{E}-04$ & 0.50 & 2.91 & 0.41 & 122.4 \\
\hline 5596 & -ELCAMS*EEQFR- & 1422.57660 & 1.42003 & 2 & CID & $9.85 \mathrm{E}-01$ & 0.01 & 1.32 & 0.32 & 115.3 \\
\hline 5729 & -LLNIT\#ADPMDWSPSNVQK- & 2011.00590 & 1.32618 & 2 & CID & $2.40 \mathrm{E}-07$ & 0.54 & 3.16 & 0.28 & 174.0 \\
\hline 5729 & -LLNITADPMDWS\#PSNVQK- & 2011.00590 & 1.32618 & 2 & CID & $5.92 \mathrm{E}-04$ & 0.05 & 2.28 & 0.37 & 116.2 \\
\hline
\end{tabular}

${ }^{1}$ SEQUEST data identifying the three phosphoacceptor sites in the pointed domain of PDEF: Thr144, Ser151, and Ser187. highlighted in red are peptides containing the Ser187 phosphoacceptor site and in green are the Thr144, and Ser151 containing peptides. A +80 amu and/or -18 amu Shift in MH+ in the phospho-peptide over the non-phosphorylated peptide confirms phosphorylation at these sites. '*'Denotes phosphorylated site identified through gain of 80 amu and '\#'denotes phosphorylated site identified through loss of 18 amu.

acids 140-157, LLNIT*ADPMWS*PSNVQK, with a mass of 2029.0059 (Table 1).

\section{Inhibition of CK2 Reduces the Steady State Level of PDEF in Prostate Cancer Cells}

Apigenin (4',5,7,-trihydroxyflavone) is a common flavonoid distributed widely in fruits and vegetables [25], and is a potent inhibitor of CK2 and several other kinases [26]. To determine if apigenin exposure affects PDEF stability in prostate cancer cells, LNCaP cells were cultured in the presence or absence of apigenin and the steady-state level of PDEF was monitored by Western blot analysis after 4 hours of exposure. Significant down-regulation of PDEF was observed in LNCaP cells after 4 hours of apigenin exposure, raising the possibility that $\mathrm{CK} 2$ blockade altered PDEF accumulation (Fig. 2A). However, while apigenin is a potent inhibitor of CK2, it has also been shown to block the activity of other kinases in vitro [26]. To further investigate the possibility that $\mathrm{CK} 2$ inhibition may affect PDEF level, LNCaP cells were treated with the highly CK2-selective inhibitor TBB [27]. After 4 hours of TBB treatment, the steady-state level of PDEF in LNCaP cells was reduced to an extent similar to that observed after apigenin treatment (Fig. 2C). Parallel Northern blots conducted on apigenin (Fig. 2A, bottom panel) and TBB-treated LNCaP cells (data not shown) revealed that the effect occurs post-transcriptionally, since PDEF mRNA accumulation was not affected by CK2 pharmacologic inhibition. In contrast, NKX3.1 has been shown to be affected at both the protein and mRNA level in similar CK2 pharmacologic inhibition studies [16]. Morphologic analysis by light microscopy revealed that approximately $10-30 \%$ of the cells lost contact with the cell culture vessel and were rounded and floating during the course of apigenin and TBB treatment. This effect was seen as early as 60 minutes after exposure to CK2 inhibitory agents.

To determine the role of both CK2 catalytic subunits in maintaining the steady-state level of PDEF, specific siRNAs were used to knock down either CK $2 \alpha$ or CK $2 \alpha^{\prime}$. In LNCaP cells transfected with a CK2 $\alpha^{\prime}$-specific siRNA, PDEF accumulation was diminished after 24 hours (Fig. 2D). In contrast, knockdown of CK2 $\alpha$ did not affect the protein level of PDEF (Fig. 2D).

\section{Blocking the 26S Proteasome Reverses the Effect of CK2 Inhibition on PDEF Accumulation}

Based on the results of pharmacologic and siRNAmediated CK2 inhibition, we hypothesized that phosphorylation by CK2 stabilized PDEF, and that the abrogation of CK2 activity resulted in PDEF degradation, possibly by the $26 \mathrm{~S}$ proteasome pathway. To determine if this was the case, LNCaP cells were treated with apigenin in the presence or absence of the proteasome inhibitor MG132. The effect of apigenin on PDEF accumulation was reversed in the presence of MG132 (Fig. 2B), suggesting that PDEF is degraded by the $26 \mathrm{~S}$ proteasome in prostate cells and that phosphorylation by $\mathrm{CK} 2$ prevents this degradation. 
A

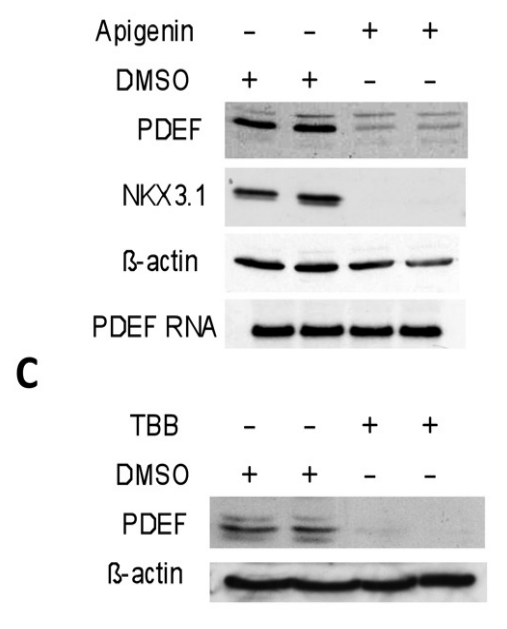

B

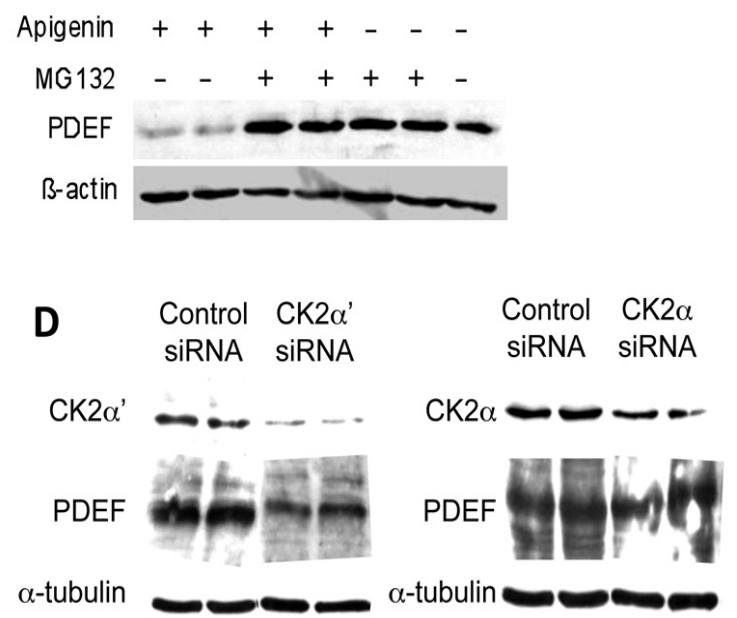

Fig. (2). Inhibiting Protein Kinase CK2 Reduces Endogenous PDEF. A. Western blot analyses (top 3 panels) to detect PDEF and NKX3.1 in LNCaP cells treated with apigenin for 4 hours. The same membrane was probed to detect $\beta$-actin as a loading control. Northern blot analysis (bottom panel) to detect PDEF mRNA. B. Western blot analysis to detect PDEF in LNCaP cells cultured for 4 hours in the presence or absence of MG132 and apigenin. MG132 treatment reverses the effect of apigenin on PDEF. C. Western blot analysis to detect PDEF in LNCaP cells treated with TBB for 4 hours. D. Western blot analysis to detect PDEF in LNCaP cells transfected with siRNA to knock down expression of individual CK2 catalytic subunits. PDEF is reduced in LNCaP cells treated with siRNA to CK2 $\alpha^{\prime}$. The blots were stripped and probed with an antibody against $\beta$-actin as a loading control.

\section{TOPORS Interacts with and Ubiquitinates PDEF}

Based on the fact that TOPORS ubiquitinates NKX3.1 in prostate cancer cells [28], we hypothesized that PDEF might also be a TOPORS substrate. To determine if TOPORS can

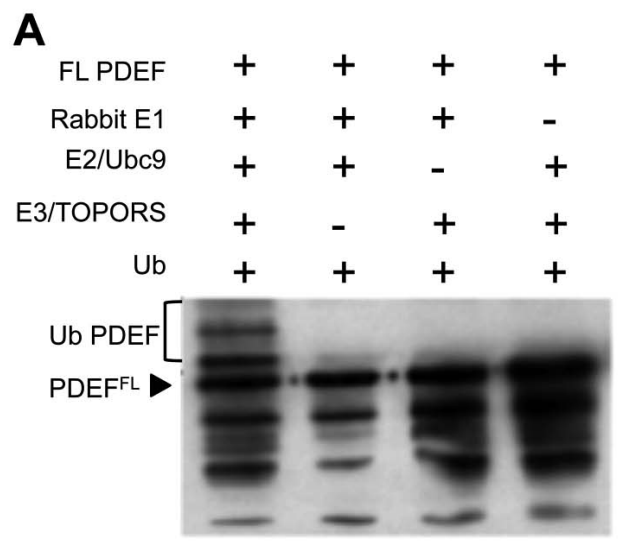

ubiquitinate PDEF, in vitro ubiquitination assays were performed in the presence of ubiquitin, E1 and UbcH5a E2 enzymes, and TOPORS (Fig. 3A). Higher molecular weight forms of PDEF were observed that were not present in the

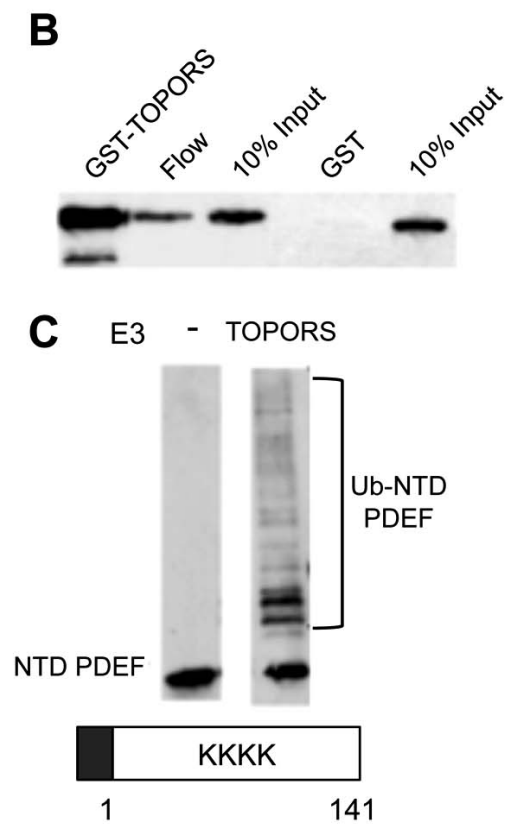

Fig. (3). PDEF interacts with and is ubiquitinated by the E3 ligase TOPORS. A. TOPORS ubiquitinates PDEF in vitro. In vitro ubiquitination assays were conducted using recombinant E1, E2 (UbcH5a), E3 (TOPORS), Ub, and PDEF. A Western blot using anti-PDEF antibodies is shown. B. Western blot of a GST pull-down assay using anti-PDEF antibodies. Equimolar amounts of GST and GST-tagged TOPORS immobilized on glutathione beads were incubated with purified His-tagged recombinant PDEF, washed, and eluted with SDSPAGE buffer. C. The N-terminal region of PDEF (PDEF ${ }^{1-141}$, NTD-PDEF) is ubiquitinated in vitro by TOPORS. A Western blot using anti$\mathrm{PDEF}$ antibodies is shown. $\mathrm{PDEF}^{\mathrm{FL}}$, full-length PDEF. 
control reactions, suggesting that TOPORS ubiquitinates PDEF in vitro. To determine if TOPORS could interact with PDEF, GST pull-down assays were performed. Recombinant GST-tagged TOPORS was purified from bacterial lysates and immobilized on glutathione beads as bait, and recombinant His-tagged PDEF was captured by TOPORS in pulldown assays (Fig. 3B).

\section{The $N$ Terminal Region of PDEF is Ubiquitinated by TOPORS}

PDEF is a 335 amino acid protein that contains 19 lysine residues, four reside in the $\mathrm{N}$ terminal region, four reside in the Pointed domain, one resides in the Pointed/ETS interdomain region, and ten reside in the ETS domain. The high molecular weight forms of PDEF observed in Fig. (3A) may represent multiply mono-ubiquitinated or poly-ubiquitinated PDEF. Additionally, TOPORS robustly ubiquitinates the Nterminal region of PDEF (amino acids 1-141), which contains four lysine residues (Fig. 3C).

\section{DISCUSSION}

The reduced expression of PDEF protein in prostate cancer cells results in increased migration and invasion and the transcriptional activation of multiple genes involved in epithelial-to-mesenchymal transition [5]. Given the potential role of this protein in regulating processes central to neoplastic progression, it is important to elucidate regulatory mechanisms that operate to maintain its steady-state level and functional activity. Evidence that PDEF may be posttranscriptionally regulated in prostate epithelial cells has been reported [12]. Translation of PDEF mRNA was shown to be inhibited specifically in several prostate cancer cases, and transfection analyses implicated the 5' and 3' untranslated regions of the PDEF mRNA in translational control. A recent analysis of PDEF mRNA and protein expression in breast cancer cell lines and patient samples has clearly elucidated a post-transcriptional control mechanism that acts regulate PDEF [29]. In this study, two miRNAs capable of targeting PDEF mRNA were identified and functionally characterized. Both miR-204 and miR-510 were shown to repress PDEF translation, and both were found to be elevated in breast cancer, providing a highly plausible mechanism whereby PDEF levels can be down-regulated in patients [29].

To date, post-translational regulatory mechanisms that regulate PDEF have not been systematically addressed, although mutation of a potential MAPK phosphoacceptor site at Threonine 50 was shown to abolish the ability of $\mathrm{PDEF}$ to induce migration or invasion of MCF-10A cells in which ErbB2, CSF-1R/CSF-1 or MEK2DD were co-expressed [6]. Since PDEF and NKX3.1 functionally associate in prostate cancer cells, we sought to determine whether PDEF was regulated by two known regulators of NKX3.1, Protein Kinase CK2 and the E3 ubiquitin ligase TOPORS. CK2 phosphorylation stabilizes NKX3.1, and prevents its degradation by the $26 \mathrm{~S}$ proteasome [16]. The data reported here demonstrate that blocking CK2 activity in prostate cancer cells also results in a sharp and sustained decrease in the steady-state level of PDEF, strongly suggesting that both proteins are coordinately regulated by $\mathrm{CK} 2$ signaling. One known function of NKX3.1 is to down-regulate PDEFmediated activation on the PSA promoter. Hence, it is possible that the coordinate regulation of these two transcriptional regulatory proteins may serve to keep PDEF activity in check through a negative feedback loop.

Unlike NKX3.1, the mRNA level of PDEF was not diminished in response to CK2 blockade, suggesting that the effect occurs exclusively at the post-translational level. It is also interesting to note that knocking down $\mathrm{CK} 2 \alpha^{\prime}$, but not CK2 $\alpha$, affected the level of PDEF. NKX3.1 also appears to be preferentially regulated by $\mathrm{CK} 2 \alpha^{\prime}$ in LNCaP cells [16]. Although the overall activity of CK2 is elevated in prostate tumor cells compared with normal prostate epithelial cells, the subcellular location and amount of free and complexed catalytic subunits of the kinase are relevant to the resulting biology [21]. The loss of the free CK $2 \alpha^{\prime}$ catalytic subunit from the nucleus could contribute to reduced expression of PDEF and NKX3.1 in prostate cancer.

In light of our observation that PDEF is readily phosphorylated in vitro by $\mathrm{CK} 2$, it is plausible that the $\mathrm{CK} 2$ stabilizing effect is direct. Mass spectrometric analyses provided evidence for three CK2 phosphoacceptor sites within the PDEF Pointed domain and the function of CK2 phosphorylation at these sites is currently under investigation. Given the importance of the Pointed domain in mediating protein-protein interactions, it will be interesting to determine how mutation of these sites affects the PDEF interactome that has recently been described [13].

Our observation that treatment with MG132 prevents the loss of PDEF when CK2 activity is blocked provides strong evidence that PDEF turnover is largely proteasome-dependent, and that at least one role of CK2 phosphorylation may be to prevent proteasome-mediated degradation. Proteasome blockade is a promising new approach to treat prostate and other forms of cancer [30] either alone or in concert with other chemotherapeutics. Although stabilizing Nuclear Factor kappa-B appears to be an important functional outcome of proteasome inhibition in cancer cells, it is possible that stabilization of PDEF and the tumor suppressor NKX3.1 may also be physiologically relevant effects of these drugs in prostate cancer. Our demonstration that PDEF is a robust target of the E3 ubiquitin ligase TOPORS raises the possibility that therapies directed at TOPORS inhibition may also stabilize PDEF in prostate cancer cells. However, it is important to note that TOPORS also appears to have a tumor suppressor role in some contexts [31].

The data reported here establish CK2 as a major regulator of PDEF accumulation in prostate epithelial cells. It will be important to determine other functional consequences of CK2 phosphorylation in PDEF, and to identify other kinases that modulate the function of this ETS protein in prostate and other cancers.

\section{REFERENCES}

[1] Sharrocks AD. The ETS-domain transcription factor family. Nat Rev Mol Cell Biol 2001; 2: 827-37.

[2] Turner DP, Watson DK. ETS transcription factors: oncogenes and tumor suppressor genes as therapeutic targets for prostate cancer. Expert Rev Anticancer Ther 2008; 8: 33-42. 
[3] Wasylyk B, Hagman J, Gutierrez-Hartmann A. Ets transcription factors: nuclear effectors of the Ras-MAP-kinase signaling pathway. Trends Biochem Sci 1998; 23: 213-6.

[4] Tootle TL, Rebay I. Post-translational modifications influence transcription factor activity: a view from the ETS superfamily. Bioessays 2005; 27: 285-98.

[5] Gu X, Zerbini LF, Otu HH, et al. Reduced PDEF expression increases invasion and expression of mesenchymal genes in prostate cancer cells. Cancer Res 2007; 67: 4219-26.

[6] Gunawardane RN, Sgroi DC, Wrobel CN, Koh E, Daley GQ, Brugge JS. Novel role for PDEF in epithelial cell migration and invasion. Cancer Res 2005; 65: 11572-80.

[7] Turner DP, Findlay VJ, Kirven AD, Moussa O, Watson DK. Global gene expression analysis identifies PDEF transcriptional networks regulating cell migration during cancer progression. Mol Biol Cell 2008; 19: 3745-57.

[8] Turner DP, Moussa O, Sauane M, Fisher PB, Watson DK. Prostatederived ETS factor is a mediator of metastatic potential through the inhibition of migration and invasion in breast cancer. Cancer Res 2007; 67: 1618-25.

[9] Feldman RJ, Sementchenko VI, Gayed M, Fraig MM, Watson DK. Pdef expression in human breast cancer is correlated with invasive potential and altered gene expression. Cancer Res 2003; 63: 462631.

[10] Moussa O, Turner DP, Feldman RJ, et al. PDEF is a negative regulator of colon cancer cell growth and migration. J Cell Biochem 2009; 108: 1389-98.

[11] Sood AK, Saxena R, Groth J, et al. Expression characteristics of prostate-derived Ets factor support a role in breast and prostate cancer progression. Hum Pathol 2007; 38: 1628-38.

[12] Nozawa M, Yomogida K, Kanno N, et al. Prostate-specific transcription factor hPSE is translated only in normal prostate epithelial cells. Cancer Res 2000; 60: 1348-52.

[13] Cho JY, Lee M, Ahn JM, et al. Proteomic analysis of a PDEF Ets transcription factor-interacting protein complex. J Proteome Res 2009; 8: 1327-37.

[14] Chen H, Nandi AK, Li X, Bieberich CJ. NKX-3.1 interacts with prostate-derived Ets factor and regulates the activity of the PSA promoter. Cancer Res 2002; 62: 338-40.

[15] Oettgen P, Finger E, Sun Z, et al. PDEF, a novel prostate epithelium-specific ets transcription factor, interacts with the androgen receptor and activates prostate-specific antigen gene expression. J Biol Chem 2000; 275: 1216-25.

[16] Li X, Guan B, Maghami S, Bieberich CJ. NKX3.1 is regulated by protein kinase CK2 in prostate tumor cells. Mol Cell Biol 2006; 26: 3008-17.
[17] Rekha N, Srinivasan N. Structural basis of regulation and substrate specificity of protein kinase CK2 deduced from the modeling of protein-protein interactions. BMC Struct Biol 2003; 3: 4.

[18] Pagano MA, Cesaro L, Meggio F, Pinna LA. Protein kinase CK2: a newcomer in the 'druggable kinome'. Biochem Soc Trans 2006; 34: 1303-6.

[19] Meggio F, Pinna LA. One-thousand-and-one substrates of protein kinase CK2? FASEB J 2003; 17: 349-68.

[20] Salvi M, Sarno S, Marin O, Meggio F, Itarte E, Pinna LA. Discrimination between the activity of protein kinase CK2 holoenzyme and its catalytic subunits. FEBS Lett 2006; 580: 3948 52.

[21] Yenice S, Davis AT, Goueli SA, Akdas A, Limas C, Ahmed K. Nuclear casein kinase 2 (CK-2) activity in human normal, benign hyperplastic, and cancerous prostate. Prostate 1994; 24: 11-6.

[22] Chen $H$, Bieberich CJ. Structural and functional analysis of domains mediating interaction between NKX-3.1 and PDEF. J Cell Biochem 2005; 94: 168-77.

[23] Rajendra R, Malegaonkar D, Pungaliya P, et al. Topors functions as an E3 ubiquitin ligase with specific E2 enzymes and ubiquitinates p53. J Biol Chem 2004; 279: 36440-4.

[24] Ghadersohi A, Sood AK. Prostate epithelium-derived Ets transcription factor mRNA is overexpressed in human breast tumors and is a candidate breast tumor marker and a breast tumor antigen. Clin Cancer Res 2001; 7: 2731-8.

[25] Chiang LC, Ng LT, Lin IC, Kuo PL, Lin CC. Anti-proliferative effect of apigenin and its apoptotic induction in human Hep G2 cells. Cancer Lett 2006; 237: 207-14.

[26] Sarno S, de Moliner E, Ruzzene M, et al. Biochemical and threedimensional-structural study of the specific inhibition of protein kinase CK2 by [5-oxo-5,6-dihydroindolo-(1,2-a)quinazolin-7yl]acetic acid (IQA). Biochem J 2003; 374: 639-46.

[27] Sarno S, Reddy H, Meggio F, et al. Selectivity of 4,5,6,7tetrabromobenzotriazole, an ATP site-directed inhibitor of protein kinase CK2 ('casein kinase-2'). FEBS Lett 2001; 496: 44-8.

[28] Guan B, Pungaliya P, Li X, et al. Ubiquitination by TOPORS regulates the prostate tumor suppressor NKX3.1. J Biol Chem 2008; 283: 4834-40.

[29] Findlay VJ, Turner DP, Moussa O, Watson DK. MicroRNAmediated inhibition of prostate-derived Ets factor messenger RNA translation affects prostate-derived Ets factor regulatory networks in human breast cancer. Cancer Res 2008; 68: 8499-506.

[30] Roccaro AM, Vacca A, Ribatti D. Bortezomib in the treatment of cancer. Recent Pat Anticancer Drug Discov 2006; 1: 397-403.

[31] Pungaliya P, Kulkarni D, Park HJ, et al. TOPORS functions as a SUMO-1 E3 ligase for chromatin-modifying proteins. J Proteome Res 2007; 6: 3918-23. 